morphogenesis. Their prevalence is estimated between 0.4 and $0.6 \%$ of live births. CHD is essentially multifactorial. Among the genetic causes, chromosomal aberrations are involved in congenital heart disease. Indeed, among patients who carry chromosomal abnormalities, $30 \%$ have cardiovascular problems. $22 \mathrm{q} 11.2$ microdeletion is the most common cause.

The purpose of this study was to determine whether subtle chromosomal anomalies previously undetected by conventional cytogenetic banding methods could be identified by array-CGH in children with isolated CHD. We reported 30 unrelated newborns recruited from Neonatology service for genetic exploration.

Genetic investigations are essentially based on the techniques of cytogenetics and molecular cytogenetics. At first intension banded karyotyping was performed followed by fluorescent in situ hybridization (FISH) using gene-specific probe TUPLE1 in 22q11.2. As a last resort comparative genomic hybridization CGH-array 44K (Agilent ${ }^{\circledR}$ Technology) has been performed for 4 patients.

FISH showed normal hybridization to the DiGeorge syndrome critical region for all patients and no copy number variations was detected by array-CGH.

Our analysis was limited by a small and heterogenous study population. Also Increasing resolution arrays are needed to detect cryptic rearrangements.

We propose this strategy to explore a wider group of patients to identify new genetic factors involved in the development of cardiac malformations. The identification of genetic etiologies for CHD is important to provide genetic counseling and to establish a report genotype phenotype for every type of heart disorder.

\section{MOLECULAR CHARACTERIZATION OF DER (8) (0TER021.13: PTERP23.3) DN IN A CHILD ASSOCIATING PSYCHOMOTOR RETARDATION, HYDROCEPHALUS AND FACIAL DYSMORPHISM}

doi:10.1136/archdischild-2012-302724.0738

1.2 $\mathrm{H}$ Hannachi, ${ }^{1,2} \mathrm{~S}$ Mougou-Zerelli, ${ }^{3} \mathrm{~K}$ Ben Helal, 1,21 Ben Abdallah, 'D Hmida, 1.2 $\mathrm{H}$ Elghezal, ${ }^{1,2 \mathrm{~A}}$ Saad. 'Cytogenetic, Biologie Moléculaire et Biologie de la Reproduction Humaines, CHU Farhat Hached; '2Département Commun de Génétique, Université de Médicine, Sousse; ${ }^{3}$ Département de Pédiatrie, Hôpital Régional Iben el Jazzar, Kairouan, Tunisia

Complex but balanced chromosomal rearrangements can give rise, through recombination during meiosis, to complex unbalanced rearrangements. Here, we report on the case of a 21 months old child associating a 8q21.13 duplication and 8p23.3 microdeletion. The proposita was referred to our lab for cytogenetic exploration of a hydrocephalus associated with facial dysmorphism. He had also psychomotor retardation and microcephaly. The patient R-banding karyotype revealed a partial trisomy $8 \mathrm{q}$ captured by the $\mathrm{p}$ telomere of the same chromosome, whereas the parents' karyotypes were normal. CGH-array technique characterized breakpoints and estimated its size to $61.8 \mathrm{Mb}$. Interestingly, an additional cryptic loss of $260 \mathrm{~Kb}$ in 8(p23.3-pter) was also identified by the same technique. These anomalies were confirmed by FISH technique.

The partial deletion of a chromosome arm in combination with partial duplication of the other was evocative of a recombinant chromosome deriving from a parental pericentric inversion. We suggest, therefore that a parental pericentric prezygotic $8(\mathrm{p} 23.3-\mathrm{q}$ 21.13) inversion resulted in the complex unbalanced rearrangement of chromosome 8 in our patient. The clinical picture including hydrocephalus, inguinal hernia, long-term fever and psychomotor retardation, was described in patients with pure 8q2-qter duplication. However, the 8p23.3 microdeletion may contribute to the psychomotor retardation, microcephaly and some minor dysmorphic features. Here we emphasize the fact that the $8 p$ microdeletion would be cytogenetically undetectable in the absence of CGH-array technique underlining the need of entire genome high-resolution analysis in patients with idiopathic mental retardation and/or birth defects even in abnormal conventional karyotype cases.

\section{MONOSOMY: GENOTYPE-PHENOTYPE CORRELATION AND THERAPEUTIC IMPACT}

doi:10.1136/archdischild-2012-302724.0739

'M Kammoun, 'H Hannechi, 'H Hajlaoui, ${ }^{2} \mathrm{H}$ Soua, 'D Hmida, 'A Saad, 'S Mougou. ${ }^{1}$ Departments of Cytogenetics and Reproductive Biology, Farhat Hached University Teaching Hospital, Sousse, 2Pediatric Department, Taher Sfar Hospital, Mahdia, Tunisia

The deletion of the long arm of chromosome 18 causes a contiguous gene deletion syndrome with a highly variable phenotype, usually related to the extent of the deleted region. The most commonly reported clinical features include: mental disabilities, decreased growth, microcephaly and facial abnormalities.

We report on a case with partial monosomy 18q22 derived from a maternal reciprocal translocation $t(8 ; 18)$. The patient was 7 months old referred for genetic exploration of neurodevelopmental delay, craniofacial dysmorphism, post natal growth retardation, choanal atresia, club foot and congenital hip dislocation.

Chromosome analysis from peripheral blood showed a 46, XY, der (18). Array CGH was performed and revealed a partial monosomy $18 \mathrm{q} 21.33 \mathrm{q} 22.31$ of $15.3 \mathrm{MB}$ associated with partial trisomy $8 \mathrm{q} 24.12 \mathrm{q} 24.23$ of $22.7 \mathrm{MB}$ region. These results were confirmed by FISH using telomeric 18q probe.

Choanal atresia and skeletal malformation are in agreement with the monosomy 18q. Interestingly, the deletion includes GALR1 gene in 18q23 witch encodes galanine receptor. Galanine is a neuromodulator that stimulates growth hormone secretion. $M B P$, and adjacent genes, are implicated in myelination process and haploinsufficiency explains partially developmental delay. Otherwise, the haploinsufficiency of the 18q22.3-q23 gene region is suggested to be a critical region for the immunoglobuline A deficiency which is significantly associated to celiac disease. Our patient has not until now immunological desorders.

The association deletion 18q22- GH deficiency and decreased myelinisation is now well estabilished. The real therapeutic impact of GH treatment is discussed.

\section{DUPLICATION OF THE SOX3 GENE IN A SRY NEGATIVE 46 XX MALE}

doi:10.1136/archdischild-2012-302724.0740

'Z Gucev, ${ }^{2} \mathrm{~F}$ Riepe, ${ }^{3} \mathrm{~A}$ Gharavi, ${ }^{3} \mathrm{~S}$ Sanna-Cherchi, 'V Tasic. 'Medical Faculty Skopje, Skopje, FYR Macedonia; ${ }^{2}$ Clinic for General Pediatrics, Kiel, Germany; ${ }^{3}$ Division of Nephrology, Columbia University, New York, NY, USA

Case presentation An 11 old patient with hypoplasia of the right kidney and hypospadias was found to be SRY negative, 46, XX. His parents and younger sister were healthy. His intelligence was normal (IO 92) and he had no other anomalies. The behavior, growth and development were all normal. His testes were $>4 \mathrm{ml}$ and the penis was $5 \mathrm{~cm}$. Ultrasound and MRI did not show internal female genitals, while confirming right kidney hypoplasia (as did the DMSA scan).

ACTH test showed normal basal and stimulated $17 \mathrm{OH}$-progesterone excluding a form of 46XX DSD due to 21-hydroxylase deficiency. 11-DOC and 11S were normal at both baseline and after ACTH stimulation, excluding 11-hydroxylase deficiency. Cortisol levels were in the mid normal range at baseline and responded to stimulation, excluding primary adrenal insufficiency. Androstenedione,

The hCG test found testosterone in the low normal range for male sex and age at baseline. It rised up to $146 \mathrm{ng} / \mathrm{mL}$ indicating the presence of functional Leydig cells targeted by hCG. The stimulated ratio T:DHT was 5.6, not supporting 5 alpha-reductase deficiency. 
SNP array for copy number variations (CNV's) showed a unique $550 \mathrm{~kb}$ duplication involving SOX3, RP1-177G6, and CDR1 genes, and the microRNA MIR320D2. This CNV was absent in 13,839 controls.

Conclusions A SRY negative 46, XX male with renal hypodysplasia was found to have an exceedingly rare duplication involving the SOX-3 gene, proving its role in sex determination and suggesting its evolvement in kidney development.

\section{PETERS PLUS SYNDROME}

doi:10.1136/archdischild-2012-302724.0741

I Maaloul, L Sfaihi, K Baklouti, S Ben Ameur, H Aloulou, T Kamoun, M Hachicha. Hedi Chaker Hospital, Sfax, Tunisia

Background Peters Plus syndrome is an autosomal recessive and rare disorder characterized by a variety of anterior eye chamber defects, of which the Peters anomaly occurs most frequently. Other major symptoms are a disproportionate short stature, developmental delay, characteristic craniofacial features, and cleft lip and/or palate.

Observations We report 4 cases of peters plus syndrome who were admitted in the department of pediatric of sfax. The family history revealed consanguineous parents in 3 cases, ocular abnormalities in 2 cases and the death of sibling in one case. Multiples abnormalities were noted in all cases just after birth like bilateral corneal opacities and facial dysmophism. 3 new borns had Skeletal system abnormalities (Short limbs, Short, broad hands, clinodactyly). Congenital heart malformations were present in 2 cases and renal anomalies were noted in one case. During the evolution, 3 patients presents a failure to thrive and developmental delay. One patient was died because of pneumonia.

Conclusion Peters plus syndrome is inherited in an autosomal recessive manner. The diagnosis is based on clinical findings and genetic analysis; prenatal diagnosis for pregnancies at increased risk are possible if the disease-causing mutations in the family are known.

\section{CHROMOSOMAL ABNORMALITIES IN A TERTIARY NEONATAL INTENSIVE CARE UNIT}

doi:10.1136/archdischild-2012-302724.0742

'A Dursun, ${ }^{2} \mathrm{~T}$ Tos, 'N Hakan, 'BS Karagol, 'N Karadag, 'B Aydin, 'A Zenciroglu, 'S Beken, 'N Okumus. 'Neonatology; 'enetic, Dr Sami Ulus Maternity and Children's Health and Diseases Training and Research Hospital, Ankara, Turkey

Background and Aim Congenital malformations and chromosomal abnormalities are important problems for the neonatal morbidity and mortality especially in well developed countries. In this study we investigated the prevalence and distribution of the chromosomal abnormalities in our NICU.

Method Chromosomal abnormalities identified between 2008 and 2012 were retrospectively analyzed. Also, demographic features and concomitant congenital malformations were also collected. Cytogenetic analysis was performed on peripheral blood of newborns by standard chromosomal analysis methods. According to our hospital protocols, newborns with more than one major malformation or with 1 major plus 2 minor abnormalities were scheduled for chromosomal abnormalities.

Results During the study period, 431 chromosomal analyses (3.2\%) were performed among the 5170 hospitalized newborns. $78.4 \%$ of the cases had normal chromosomal karyotype. Consanguinity rate was $27.9 \%$. Major chromosomal abnormality rate was $1.8 \%$.

Frequency of chromosomal abnormalities were as follows; Trisomy $2113.5 \%(n=58)$, trisomy $182.3 \%(n=10)$, trisomy $131.2 \%$ $(n=5), 45 \mathrm{XO} 0.5 \%(n=2)$ and other chromosomal abnormalities were $4.2 \%(n=18)$. Congenital heart abnormalities (85.9\%), craniofascial abnormalities (44.6\%) and genito-urinary anomalies (16.9\%) were most common concomittant malformations. Cardiovascular abnormalities were most common malformations in newborns with trisomy 21.

Conclusion Frequency and distribution of the chromosomal abnormalities in our NICU were similar compering with other populational studies. Trisomy 21 was most common chromosomal abnormality. Newborns with malformations in more than two organ system should be investigated chromosomally as well.

\section{LYSOSOMAL STORAGE DISORDERS IN NON- IMMUNOLOGICAL HYDROPS FETALIS - MORE COMMON THAN ASSUMED?}

doi:10.1136/archdischild-2012-302724.0743

${ }^{1} \mathrm{C}$ Whybra, ${ }^{2} \mathrm{E}$ Mengel, ${ }^{3} \mathrm{~F}$ Bahlmann, ${ }^{4} \mathrm{C}$ Kampmann, ${ }^{2} \mathrm{M}$ Beck, ${ }^{1} \mathrm{E}$ Mildenberger. ${ }^{1}$ Neonatology; ' 2 Department of Lysosomal Storage Disorder, Villa Metabolica, University Medical Center of the Johannes Gutenberg University, Mainz; ${ }^{3}$ Department of Obstetrics and Gynecology, Buergerhospital, Frankfurt/Main; ${ }^{4}$ Department of Pediatric Cardiology, Medical Center of the Johannes Gutenberg University, Mainz, Germany

Background Although non immunological hydrops fetalis (NIHF) is a very rare disorder, the disturbance accounts for a disproportionate share $(3 \%)$ of overall mortality in the perinatal period. Lysosomal storage disorders (LSD) are only exceptionally considered to be the cause of NIHF. The reported incidence is about $1 \%$. On the other hand, in about $18 \%$ of all cases, NIHF is classified as idiopathic.

Patients and methods We report four cases of transient NIHF due to LSD and reviewed the literature for LSD associated with NIHF.

Results At present, 12 different LSD are described to be associated with NIHF. The majority of reported patients already had a family history of NIHF, which had not been investigated. A diagnostic approach to the fetus with NIHF due to suspected LSD is suggested.

Conclusions Extensive and thorough investigation of the etiology of NIHF is obligatory. In particular, LSD should be considered in idiopathic NIHF. Enzymatic studies in chorionic villous samples or amniotic cultured cells, once the most common conditions associated with NIHF have been ruled out, should be performed. We assume that the incidence of LSD in NIHF is significantly higher than the estimated $1 \%$ reported in previous studies. This is important for genetic counseling, as there is at first, a high risk of recurrence and, secondly, the availability of enzyme replacement therapy for an increasing number of LSD.

\section{CHOLESTEROL ESTER TRANSFER PROTEIN GENE POLYMORPHISM AND SELECTED LIPIDS PARAMETERS IN CHILDREN FROM FAMILIES WITH HISTORY OF CVS DISEASES}

doi:10.1136/archdischild-2012-302724.0744

E Pac-KoŻuchowska, A Mroczkowska-Juchkiewicz, A Pawłowska-Kamieniak, K Kominek, D Gołyska. Medical University, Lublin, Poland

The human population especially predisposed to early development of atherosclerosis are children from families with history of cardiovascular system diseases. The aim of this study was to examine lipids parameters associated with cardiovascular diseases and polymorphisms of G279A located in the Cholesterol Ester Transfer Protein (CETP) gene.

Material/methods The study covered 30 children aged 5-6 years from families with history of cardiovascular system diseases. The children were examined physically, and nutritional status assessed. In all of the children examined, the blood concentrations of 